\title{
Aneurysmal bone cyst secondary to a giant cell tumor of the patella: A case report
}

\author{
XIAOLONG YU ${ }^{1 *}$, RUNSHENG GUO ${ }^{2 *}$, CONGLIANG FAN ${ }^{1}$, HUCHENG LIU ${ }^{2}$, \\ BIN ZHANG $^{1}$, TAO NIE ${ }^{1}$, YI TU ${ }^{3}$ and MIN DAI ${ }^{1}$ \\ ${ }^{1}$ Department of Orthopedics, The First Affiliated Hospital of Nanchang University, \\ Artificial Joints Engineering and Technology Research Center of Jiangxi Province; \\ ${ }^{2}$ Multidisciplinary Therapy Center of Musculoskeletal Tumor; ${ }^{3}$ Department of Pathology, \\ The First Affiliated Hospital of Nanchang University, Nanchang, Jiangxi 330006, P.R. China
}

Received February 11, 2015; Accepted October 21, 2015

DOI: $10.3892 / 01.2016 .4080$

\begin{abstract}
The patella is an unusual location for primary and metastatic bone tumors to develop. The most frequently encountered primary osteolytic lesions at the patella include giant cell tumors of the bone (GCT), chondroblastoma and aneurysmal bone cysts (ABC). However, the presentation of an $\mathrm{ABC}$ originating secondary to a GCT at the patella is rare. The present study describes such a case in a 46-year-old female. The differential diagnosis of the condition was extensive. The patient underwent curettage and the addition of bone cement to fill the defect. Pathological analysis of the resected tissue demonstrated that the lesion was consistent with an ABC forming secondary to a GCT. A 3-month follow-up was completed subsequent to the surgery, with a computed tomography scan demonstrating no evidence of recurrence. However, frequent and continuous observations of the patient following diagnosis are planned in order to evaluate the long-term efficacy of the surgical treatment. To the best of our knowledge, the present study describes the third reported case in the literature of this rare, double synchronous, benign tumor located at the patella.
\end{abstract}

\section{Introduction}

A giant cell tumor of the bone (GCT) is a locally aggressive, benign neoplasm that consists of sheets of neoplastic mononuclear cells interspersed amongst non-neoplastic,

Correspondence to: Mr. Hucheng Liu, Multidisciplinary Therapy Center of Musculoskeletal Tumor, The First Affiliated Hospital of Nanchang University, 17 Yong Wai Zheng Street, Nanchang, Jiangxi 330006, P.R. China

E-mail: 1207672246@qq.com

*Contributed equally

Key words: giant cell tumor of the bone, aneurysmal bone cyst, patella, surgery, pathology, imaging, differential diagnosis uniformly distributed, osteoclast-like giant cells (1). The tumor frequently arises from the metaphysis of the long bones in adults aged between 30 and 50 years, with epiphyseal destruction and growth towards the sub-articular region (1). GCTs account for $~ 4-9.5 \%$ of all primary bone tumors (2). An aneurysmal bone cyst (ABC) is a benign, expansile, osteolytic lesion that presents as an aggressive, vascular disorder in the bones. The cyst is an erratic and expansive growth, depicted by a 'soap bubble' appearance on radiographs. The numerous blood-filled cavities experience pressure, with marked ballooning of a thinned cortex (3). An ABC typically develops in the long bones and spine of children and young adults (3). $\mathrm{ABC}$ was first described as a distinct entity in 1942 by Jaffe and Lichtenstein (4). Primary ABCs account for $1.4 \%$ of all primary bone tumors (5). In $\sim 70 \%$ of cases, ABCs will arise as primary tumors without any recognized antecedent bone lesion, however, in $\sim 30 \%$ of cases, a pre-existing osseous lesion can be identified (6). GCTs are the most prevalent precursor lesions of $\mathrm{ABCs}$ developing secondarily (6). The present study describes the case of an ABC forming secondary to a GCT at the patella in a 46-year-old female patient.

\section{Case report}

In December 2014, a 46-year-old female attended the Orthopedic Oncology Clinic at The First Affiliated Hospital of Nanchang University (Nanchang, China) due to increasing local pain and swelling in the right knee. The patient stated that these symptoms had arisen 6 months previously, but had intensified during the last 2 months prior to admittance. The patient recalled a previous right knee injury that had occurred 6 months ago, but no other physical problems were reported and it was established that the patient had a positive family history of bone neoplasms. There was no fever or respiratory embarrassment accompanying the pain and mass. Furthermore, no history of weight loss or exposure to tuberculosis was specified. During the initial physical examination, the passive and active range of motion of the right knee joint was limited. The overall neurocirculatory status was normal, as was muscle-tendon strength, and the patient did not exhibit anatomical deformity of the right lower extremity. During the 

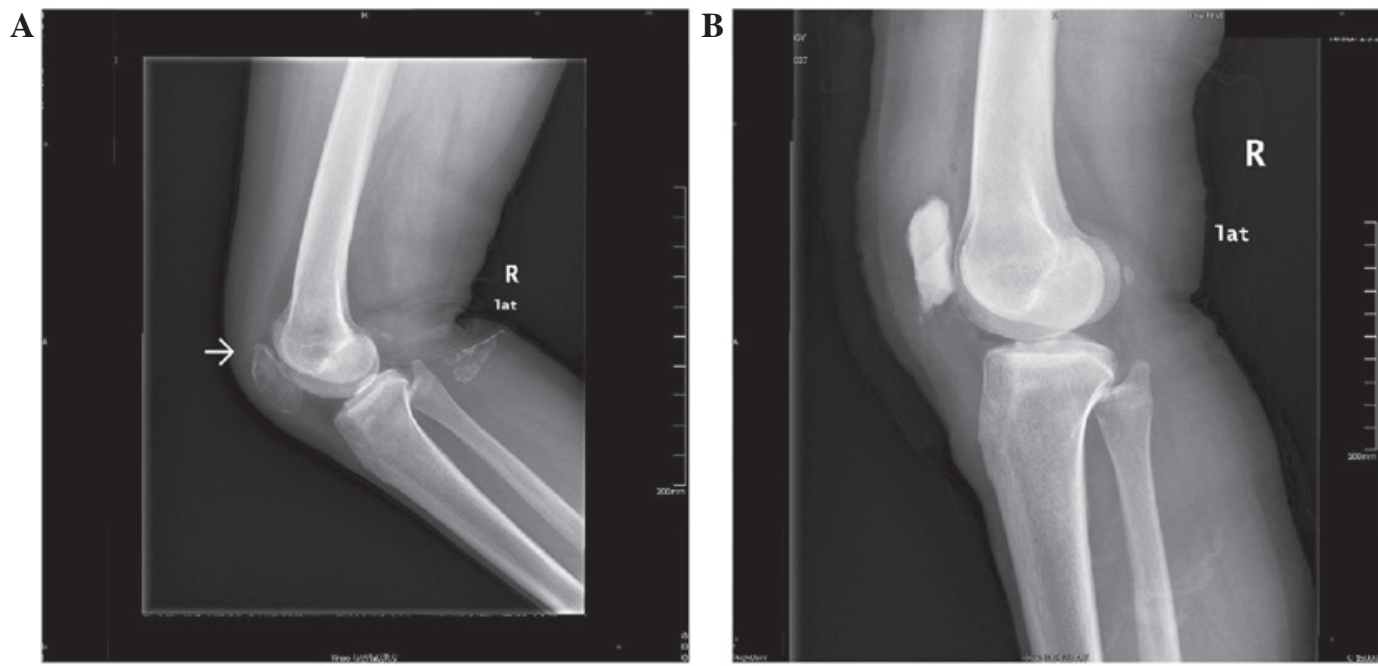

Figure 1. Pre-operative and post-operative standard knee radiographs. (A) Right knee radiograph in lateral view demonstrating a multiseptated expansile lytic lesion in the patella, with a cortical break (arrow) in the supra-patellar region suggesting a pathological fracture. Bony structures of the adjacent femur and tibia, and an extraosseous soft-tissue lesion are not present. (B) Correct right patella shape following surgical curettage and cement filling.
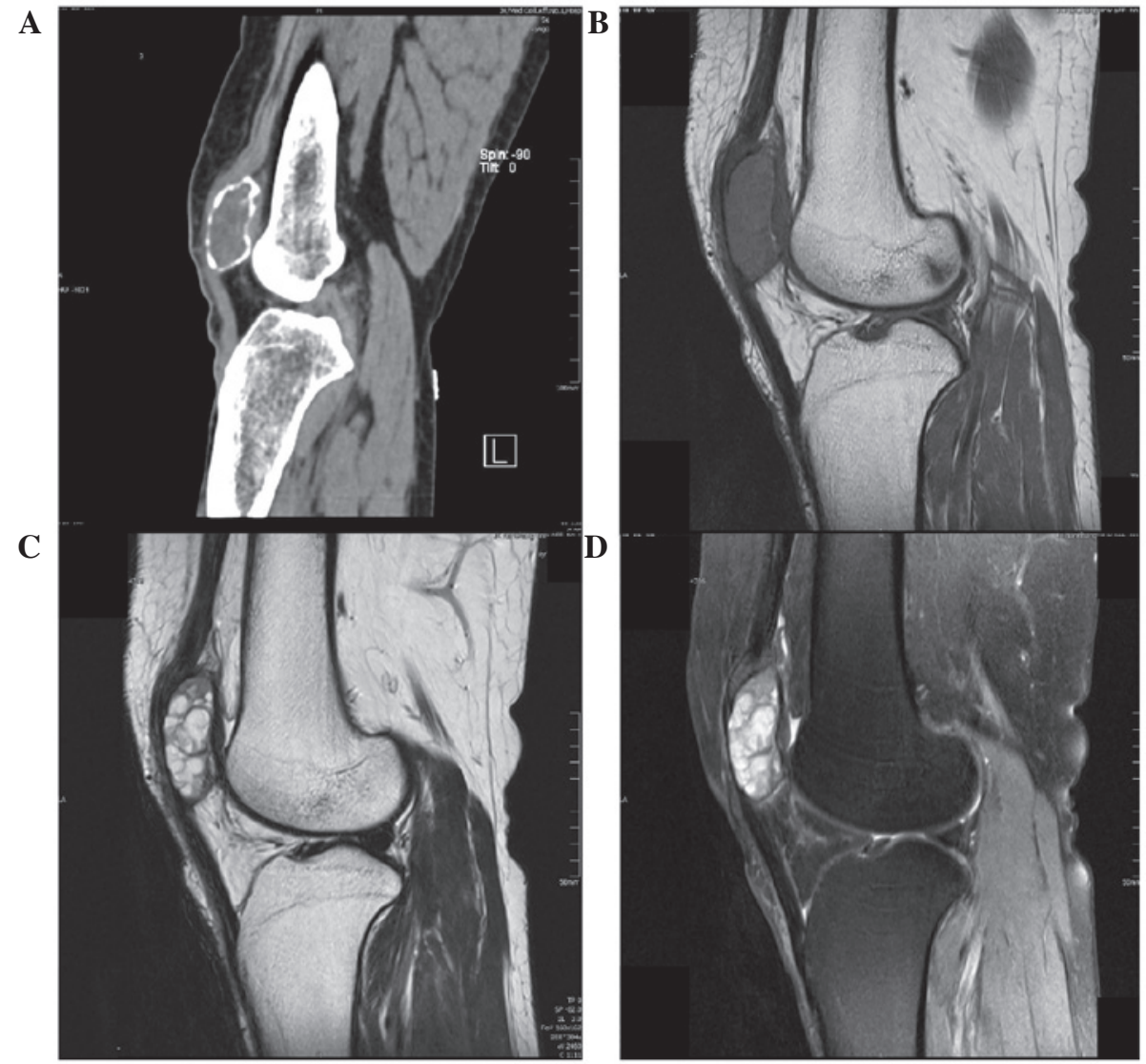

Figure 2. Pre-operative CT and MRI examination. CT scan exhibiting a multiloculated lytic lesion in the entire right patella. (A) More marked cortical thinning with a 'balloon' appearance in comparison to the X-ray, but with no significant periosteal reaction. The lesion is measured at $\sim 4 \times 3.2 \times 2 \mathrm{~cm}^{3}$ in size (B and C) Axial T1-weighted images demonstrating low signal intensity and axial T2-weighted images indicating multiple cystic lesions of varying sizes with high signal intensity, and significant multiple liquid-liquid levels. (D) T2-weighted with fat suppression also exhibiting high signal intensity. CT, computed tomography; MRI, magnetic resonance imaging.

advanced physical examination, the anterior aspect of the right knee was swollen, and the patella appeared larger and more prominent on the right side in comparison to the left. There was no localized warmth and pulsation, but the patella was particularly tender, with palpable bony thickening. There was also no synovial thickening or knee joint effusion observed. The floating patella test was negative and there was no ligamentous laxity.

Additionally, a series of laboratory tests were performed and determined that inflammatory and tumor markers fell 

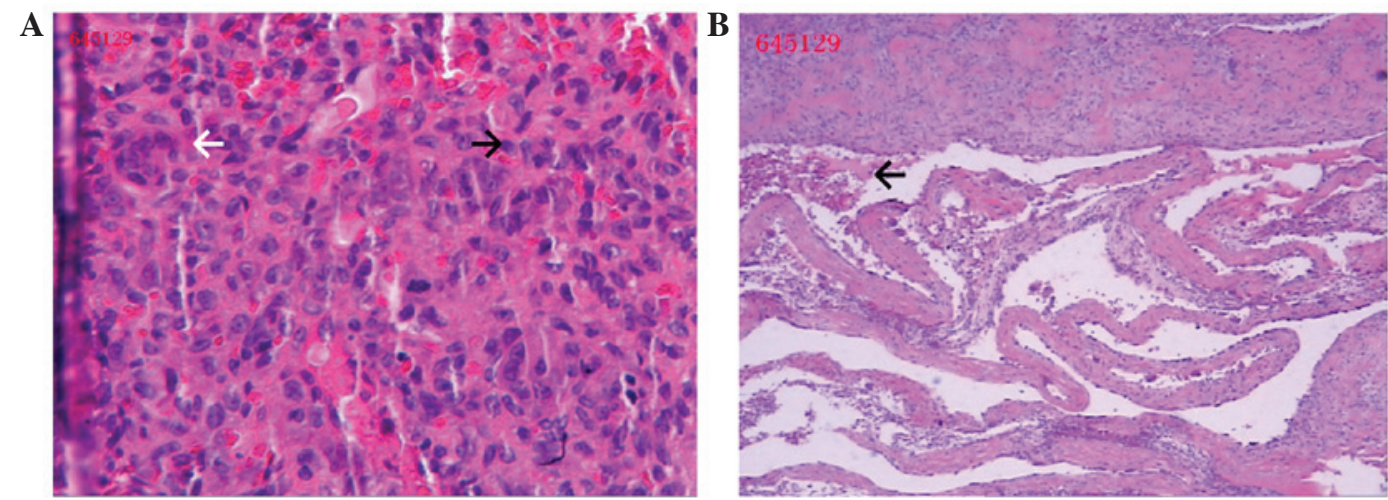

Figure 3. Post-operative histological observations. (A) Solid giant cell tumor components with mononuclear cells interspersed between multinucleated giant cells (white arrow) and mononuclear cells (black arrow) (HE staining; magnification, x200). (B) Cystic aneurysmal components with multiple blood-filled cystic spaces separated by fibrous tissue. Blood components are also present (black arrow) (HE staining; magnification, x50). HE, hematoxylin and eosin.

within the normal ranges [erythrocyte sedimentation rate, $18 \mathrm{~mm} / \mathrm{h}$ (normal range, $0-20 \mathrm{~mm} / \mathrm{h}$ ); C-reactive protein, negative] with the exception of alkaline phosphatase (179 U/l; normal range, 50-135 U/l).

Standard knee radiographs exhibited a lytic, expansile lesion with a well-defined sclerotic margin located at the right patella, which became enlarged and transformative (Fig. 1). However, the adjacent femur and tibia were normal in shape and without bony structures or deformation (Fig. 1A). Computed tomography (CT) scans indicated a multiloculated, lytic lesion in the entire right patella, particularly in the medial region (Fig. 2). Severe cortical thinning was observed in a diffuse pattern, leaving the cortex as a thin shell with a 'balloon' appearance, but no significant periosteal reaction was detected. The expansive lesion contained few radiopaque calcified bodies, and multiple liquid-liquid levels were identified (Fig. 2A). The lesion was measured at $\sim 4 \times 3.2 \times 2 \mathrm{~cm}^{3}$ in size. In accordance with the findings detected on the radiographs and CT scans, GCT, giant cell reparative granuloma and telangiectatic osteosarcoma were included in the differential diagnosis. Therefore, magnetic resonance imaging (MRI) was performed to investigate the expansive lesion further. Axial T1-weighted images exhibited low signal intensity and axial T2-weighted images indicated the presence of multiple cystic lesions of varying sizes with high signal intensities (Fig. 2B and C). In addition, multiple liquid-liquid levels were observed within the lesion on $\mathrm{T} 2$-weighted sequences and $\mathrm{T} 2$-weighted images with fat suppression also exhibited high signal intensities (Fig. 2D).

Based on the exclusion of surgical contraindications, surgeons specializing in the treatment of bone tumors performed the surgery. The patient was placed in a supine position, and following epidural anesthesia, the right knee was covered with disinfecting and paving sterile drapes, and positioned to expose the surgical field. The right lower extremity was exsanguinated and tourniquet ischemia was applied with an elastic bandage. A linear incision of $8 \mathrm{~cm}$ was formed overlying the medial right patella. The separation of tissue layers, including subcutaneous tissue, superficial fascia and deep fascia, was conducted until a thin, but unbroken, periosteal shell was identified. However, expansive invasion of the surrounding soft tissue was not observed. The cortical structure had become attenuated enough to deform with slight pressure from a finger. Curettage of the lesion was performed, with the patella subsequently filled with bone cement and closed with a tension band wire. Intraoperative tissues were extracted for pathological examination. A wound drainage tube was inserted and each layer of tissue was sutured in order to achieve complete hemostasis. The estimated total loss of blood was $\sim 300 \mathrm{ml}$, and no blood transfusion was required during the treatment process.

Histologically, the tumor tissue fragments consisted of neoplastic cell sheets with ill-defined cytoplasm, representing multinucleated giant cells that were interspersed with uniformly distributed, osteoclast-like giant cells (Fig. 3A). Furthermore, there was a second component that presented with cavernous spaces. The cavity was composed of multiple blood-filled cystic spaces, separated by fibrous tissue that contained reactive bone bordered by osteoblasts (Fig. 3B). The walls of these spaces lacked an endothelial lining, and anaplasia was not detected. The diagnosis of ABC secondary to GCT was confirmed on the basis of these histological findings.

The patient was discharged without any complications at 1 week post-surgery. At the 3-month follow-up, the patient reported no pain during daily activities and had started back at work. Furthermore, a normal range of motion of the right knee joint had been achieved. There has since been no evidence of tumor recurrence following the surgery.

The present report was approved by the Ethical Review Committee of The First Affiliated Hospital of Nanchang University, and written informed consent was obtained from the patient.

\section{Discussion}

As the largest sesamoid bone in the body, the patella is an infrequent site for primary and metastatic bone tumors to occur. Primary intraosseous tumors of the patella are extremely rare and account for $\sim 0.12 \%$ of all primary bone tumors $(7,8)$. The majority of patella tumors are benign, including chondroblastoma, GCT and ABC, with the latter two lesions being the most frequently diagnosed. These tumors are typically identified in patients $<40$ years (9). Other differential diagnoses of 
a patella lesion may include tuberculosis, lymphoma, Paget's disease, chondrosarcoma and a solitary bone cyst (10).

A GCT is a bone tumor that is characterized by the presence of multinucleated giant cells. The majority are benign, with a risk of malignancy observed in $<1 \%$ of cases (11). The majority of GCTs occur in skeletally mature patients, typically between the ages of 20 and 50 years (12). GCTs frequently arise from the metaphysis of the long bones, with epiphyseal involvement, and they typically involve either the distal end of the femur or the proximal tibia. However, $<1 \%$ of all GCTs in the skeletal system arise from the patella (13). Radiographically, the tumors demonstrate erratic and lytic lesions with a 'soap bubble' appearance and a lack of internal calcification. Cortical thinning, cortical penetration (33-50\%) with soft tissue extension, pathological, fractures (11-37\%) and a periosteal reaction (10-30\%) may also be observed $(14,15)$. MRI demonstrates low to intermediate signal intensities on T1-weighted sequences and heterogeneously high signal intensities on T2-weighted sequences (16). The radiographic features of GCTs located at the patella are similar to those positioned at other areas of the skeleton.

By contrast, $\mathrm{ABC}$ is a benign, expansile, cystic bony lesion that is composed of blood-filled spaces segregated by connective tissue septa and containing osteoclast-type giant cells, fibroblasts and reactive woven bone. ABC often develops during the second decade of life, with a slightly increased tendency for occurrence observed in females. The long bones are involved most frequently, and the spine is affected in $30 \%$ of patients (17). The lesion either occurs de novo, known as primary $\mathrm{ABC}$, or develops secondarily to a pre-existing bone lesion or trauma, known as secondary ABC. Secondary $\mathrm{ABC}$ s can be associated with GCTs, chondroblastoma, osteoblastoma, hemangioma, non-ossifying fibroma, fibrous dysplasia, eosinophilic granuloma and osteosarcoma (18). Primary ABCs account for $1.4 \%$ of all primary bone tumors, with the occurrence of a primary $\mathrm{ABC}$ located at the patella proving extremely rare. In total, $30-50 \%$ of all $\mathrm{ABC}$ are secondary (19). Two theories have been proposed regarding the development of secondary ABCs: A vascular anomaly in a primary bone lesion or a reactive bone growth leading to a hemodynamic change (20). There are existing data that suggest a neoplastic origin with chromosomal abnormalities (21-24). Despite such data and research, the etiology and pathogenesis of this rare, benign, vascular, destructive and multicystic lesion remain unclear.

In $\sim 79 \%$ of cases, ABCs arise as primary tumors without any recognized antecedent bone lesion, however, in $\sim 30 \%$ of cases, a pre-existing osseous lesion can be observed. GCTs are the most prevalent precursor lesions in terms of ABC developing secondarily $(7,25)$.

Imaging assessments, particularly MRI, are important in order to conduct a differential diagnosis. In MRI, ABCs typically have an expansile appearance with the presence of multiseptated cysts. MRI is able to identify the characteristic fluid-fluid levels, as well as demonstrate the presence of a solid component, indicating that the $\mathrm{ABC}$ is in fact secondary. In the cysts, the settling of degraded blood products gradually forms the fluid levels (26). It is important to note that the presence of fluid-fluid levels, although characteristic of $\mathrm{ABC}$, is by no means unique to it. Fluid-fluid levels are also observed in other benign and malignant lesions, including GCT, chondroblastoma, simple bone cysts and telangiectatic osteosarcoma (27). Furthermore, the thin, well-defined margins of ABCs should aid their discrimination from these other lesions. However, only $20 \%$ of secondary ABCs have the typical radiological appearance, whilst in the other $80 \%$, the associated lesion determines the radiological appearance, particularly when it is malignant. Therefore, histopathological confirmation is crucial in forming a diagnosis. However, these results must be interpreted with great care, since confusion regarding the diagnosis may still occur if the tissue sample is taken from a small biopsy or a limited sample (28-30). In the present study, the exact diagnosis of GCT with secondary ABC was only determined after histopathological evaluation of the entire tumor. Fortunately, the presence of a secondary $\mathrm{ABC}$ has no significant implication on the overall management or prognosis of the GCT. However, when the associated pre-existing lesion is malignant, the management and treatment requires adjusting accordingly (31).

In conclusion, it is important to highlight that the associations between imaging and histopathological findings are important. With regards to the limitations of a small biopsy sample, which may demonstrate features of an $\mathrm{ABC}$ but not the underlying lesion, careful review of the imaging appearance is imperative to exclude the potential of an ABC that may be secondary to a malignant lesion (30). Surgical curettage and cement filling are considered as the preferred treatment to relieve pain, improve the range of motion of the right knee joint and confirm the diagnosis histologically. A patellectomy is not recommended until a severely aggressive lesion leads to overall destruction of the patella. With regards to the present case, the patient remained symptom-free 3 months after surgery. Due to the risk of recurrence, and additional risk of malignancy, long-term follow-ups should be continued, with particular attention concerning any change in malignancy.

\section{Acknowledgements}

This study was supported by the Gan-Po Talents Project 555 of Jiangxi Province and the Support Plan of Science and Technology Department of Jiangxi Province (grant no. 20112BBG70020).

\section{References}

1. van der Heijden L, Dijkstra PD, van de Sande MA, Kroep JR, Nout RA, van Rijswijk CS, Bovée JV, Hogendoorn PC and Gelderblom H: The clinical approach toward giant cell tumor of bone. Oncologist 19: 550-561, 2014.

2. Murphey MD, Nomikos GC, Flemming DJ, Gannon FH, Temple HT and Kransdorf MJ: From the archives of AFIP. Imaging of giant cell tumor and giant cell reparative granuloma of bone: Radiologic-pathologic correlation. Radiographics 21: 1283-1309, 2001

3. Salunke P, Futane $S$ and Jain V: Aneurysmal bone cyst of cervical spine: True $360^{\circ}$ resection with emphasis on lateral masses. Neurol India 60: 113-115, 2012.

4. Jaffe HL and Lichtenstein L: Solitary unicameral bone cyst with emphasis on the roentgen picture, the pathologic appearance and the pathogenesis. Arch Surg 44: 1004-1025, 1942.

5. Oh JH, Kim HH, Gong HS, Lee SL, Kim JY and Kim WS: Primary aneurysmal bone cyst of the patella: A case report. J Orthop Surg (Hong Kong) 15: 234-237, 2007.

6. Cottalorda J and Bourelle S: Modern concepts of primary aneurysmal bone cyst. Arch Orthop Trauma Surg 127: 105-114, 2007.

7. Singh J, James SL, Kroon HM, Woertler K, Anderson SE, Jundt $G$ and Davies AM: Tumour and tumour-like lesions of the patella - a multicentre experience. Eur Radiol 19: 701-712, 2009. 
8. Mercuri M and Casadei R: Patellar tumors. Clin Orthop Relat Res 389: 35-46, 2001.

9. Saglik Y, Yildiz Y, Basarir K, Tezen E and Güner D: Tumours and tumour-like lesions of the patella: A report of eight cases. Acta Orthop Belg 74: 391-396, 2008.

10. Macdonald D, Fornasier V and Cameron J: Multicentric giant cell tumour involving the patella. Can J Surg 44: 222-223, 2001.

11. Chakarun CJ, Forrester DM, Gottsegen CJ, Patel DB, White EA and Matcuk GR Jr: Giant cell tumor of bone: Review, mimics, and new developments in treatment. Radiographics 33: 197-211, 2013

12. Campanacci M, Baldini N, Boriani S and Sudanese A: Giant-cell tumor of bone. J Bone Joint Surg Am 69: 106-114, 1987.

13. Nahal A, Ajlan A, Alcindor T and Turcotte R: Dedifferentiated giant cell tumour of bone in the form of low-grade fibroblastic osteogenic sarcoma: Case report of a unique presentation with follow-up. Curr Oncol 17: 71-76, 2010.

14. Mooren JJ, Theunissen PH and Zijlker TD: Pathology quiz case 2: Laryngeal giant cell tumor (LGCT). JAMA Otolaryngol Head Neck Surg 139: 89-90, 2013.

15. Murphey MD, Nomikos GC, Flemming DJ, Gannon FH, Temple HT and Kransdorf MJ: From the archives of AFIP. Imaging of giant cell tumor and giant cell reparative granuloma of bone: Radiologic-pathologic correlation. Radiographics 21: $1283-1309,2001$

16. Lee MJ, Sallomi DF, Munk PL, Janzen DL, Connell DG, O'Connell JX, Logan PM and Masri BA: Pictorial review: Giant cell tumours of bone. Clin Radiol 53: 481-489, 1998

17. Boriani S, Bandiera S, Casadei R, Boriani L, Donthineni R, Gasbarrini A, Pignotti E, Biagini R and Schwab JH: Giant cell tumor of the mobile spine: A review of 49 cases. Spine (Phila Pa 1976) 37: E37-E45, 2012.

18. Mankin HJ, Hornicek FJ, Ortiz-Cruz E, Villafuerte J and Gebhardt MC: Aneurysmal bone cyst: A review of 150 patients. J Clin Oncol 23: 6756-6762, 2005.

19. Wu Z, Yang X, Xiao J, Feng D, Huang Q, Zheng W, Huang W and Zhou Z: Aneurysmal bone cyst secondary to giant cell tumor of the mobile spine: A report of 11 cases. Spine (Phila Pa 1976) 36 E1385-E1390, 2011.

20. Saccomanni B: Aneurysmal bone cyst of spine: A review of literature. Arch Orthop Trauma Surg 128: 1145-1147, 2008.
21. Panoutsakopoulos G, Pandis N, Kyriazoglou I, Gustafson P, Mertens F and Mandahl N: Recurrent t(16;17)(q22;p13) in aneurysmal bone cysts. Genes Chromosomes Cancer 26: 265-266, 1999.

22. Oliveira AM, Hsi BL, Weremowicz S, Rosenberg AE, Dal Cin $P$, Joseph N, Bridge JA, Perez-Atayde AR and Fletcher JA: USP6 (Tre2) fusion oncogenes in aneurysmal bone cyst. Cancer Res 64: 1920-1923, 2004

23. Kenney B, Richkind KE and Zambrano E: Solid variant of aneurysmal bone cyst with a novel $(\mathrm{X} ; 9)$ translocation. Cancer Genet Cytogenet 178: 155-159, 2007.

24. Baruffi MR, Neto JB, Barbieri CH and Casartelli C: Aneurysmal bone cyst with chromosomal changes involving $7 \mathrm{q}$ and $16 \mathrm{p}$. Cancer Genet Cytogenet 129: 177-180, 2001.

25. Hao Y, Wang L, Yan M, Jin F, Ge S and Dai K: Soft tissue aneurysmal bone cyst in a 10-year-old girl. Oncol Lett 3: 545-548, 2012.

26. Mahnken AH, Nolte-Ernsting CC, Wildberger JE, Heussen N, Adam G, Wirtz DC, Piroth W, Bücker A, Biesterfeld S, Haage P and Günther RW: Aneurysmal bone cyst: Value of MR imaging and conventional radiography. Eur Radiol 13: 1118-1124, 2003.

27. Roemer FW, Remplik P and Bohndorf K: Uncommon aneurysmal bone cyst: Radiographic and MRI findings. AJR Am J Roentgenol 184: 349, 2005

28. Lin SP, Fang YC, Chu DC, Chang YC and Hsu CI: Characteristics of cranial aneurysmal bone cyst on computed tomography and magnetic resonance imaging. J Formos Med Assoc 106: 255-259, 2007

29. Yang BT, Wang YZ, Wang XY and Wang ZC: Imaging study of ossifying fibroma with associated aneurysmal bone cyst in the paranasal sinus. Eur J Radiol 81: 3450-3455, 2012.

30. Benamou J, Lussier B, Alexander K, Gains MJ and Savard C: Use of magnetic resonance imaging and histopathologic findings for diagnosis of an aneurysmal bone cyst in the scapula of a cat. J Am Vet Med Assoc 240: 69-74, 2012.

31. Hong WS, Sung MS, Kim JH, Kim HM, Kim TK, Chung MH, Lim YS and Lim HW: Giant cell tumor with secondary aneurysmal bone cyst: A unique presentation with an ossified extraosseous soft tissue mass. Skeletal Radiol 42: 1605-1610, 2013. 